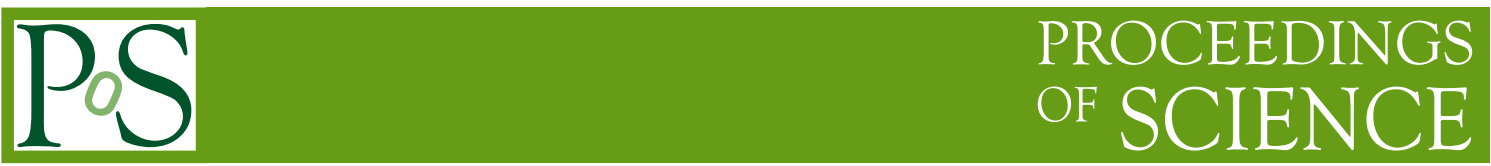

\title{
Exploiting high-energy polarization in blazars
}

\author{
Lenté Dreyer* \\ Centre for Space Research, North-West University, Potchefstroom, 2520, South Africa \\ E-mail: lentedreyer@gmail.com \\ Markus Böttcher ${ }^{\dagger}$ \\ Centre for Space Research, North-West University, Potchefstroom, 2520, South Africa \\ E-mail: Markus.Bottcher@nwu.ac.za
}

The spectral energy distributions (SEDs) and variability of astrophysical sources can be modeled in many different ways, in particular, the production of $X$-rays and $\gamma$-rays in the SEDs of blazars. High-energy polarimetry can probe the most active jet regions with the most efficient particle accelerations, and thus serve as a powerful tool to distinguish between models. We summarize the scientific potential and model predictions for high-energy polarization of blazar jets. The advantages of using Monte Carlo methods to study high-energy polarization from anisotropic Compton scattering, by thermal and non-thermal particles, will be discussed. This will reinforce the use of measurements of polarization signatures to suggest observational strategies in future projects and to use polarization as a diagnostic to distinguish between leptonic and hadronic highenergy emission from blazars.

High Energy Astrophysics in Southern Africa - HEASA2018

1-3 August, 2018

Parys, Free State, South Africa

\footnotetext{
${ }^{*}$ Speaker.

$\dagger$ The work of Markus Böttcher is supported through the South African Research Chair Initiative (SARChI) of the South African National Research Foundation (NRF) and the Department of Science and Technology (DST), under SARChI Chair grant no. 64789. Disclaimer: Any opinion, finding and conclusion or recommendation expressed in this material is that of the authors and the NRF does not accept any liability in this regard.
} 


\section{Introduction}

Active Galactic Nuclei (AGNs) are some of the most luminous objects in the universe, often observed to host relativistic jets where the bulk energy is converted to kinetic energy of electrons, multi-wavelength radiation and, possibly, particle emission from ions and neutrinos. Therefore, these particles, and the radiation across the electromagnetic spectrum, are the messengers of the mysterious astrophysical conditions in the core of the active galaxies and their jets. Blazars are some of the most extreme classes of AGNs - consisting of BL Lac objects and Flat Spectrum Radio Quasars (FSRQs) - where the observer's line of sight is closely aligned with the jet's axis. They are known to emit non-thermal dominated radiation across the entire electromagnetic spectrum, are variable at all timescales, and are characterized by high and variable polarization [1].

Various properties of the radiation from blazars have been studied with multi-wavelength observations and spectral fitting, without regarding the magnetic field evolution. Measurements of synchrotron polarization and optical emission from relativistic jet sources have been a standard way of assessing the degree of order and direction of the magnetic fields. Optical polarization, however, can often originate in regions that do not emit strong high-energy radiation and, therefore, provides no clear view of the polarization signatures in the most active acceleration regions. High-energy polarization can thus be used as a powerful tool to probe the jet physics in the most active acceleration regions with the most energetic particles [2][3].

Polarization in high-energy emission has remained largely unexplored, and no high-energy polarimetric data exists for blazars. This is partly due to the difficulties in the detection of the polarization in $X$-ray and $\gamma$-ray emission [4]. However, the $\mathrm{X}$-ray and soft $\gamma$-ray polarimeters which are at various stages of planning, design, and operation (e.g. XIPE [5], X-Calibur [6], e-ASTROGRAM [7], IXPE [8], and AMEGO [9]) have justified renewed interest in high-energy polarization. Highenergy polarimetry will give a better multi-wavelength coverage, and enable us to obtain light curves and polarization signatures from radio, all the way to $\gamma$ rays [3][4].

The origin of the high-energy component in spectral energy distributions (SEDs) of blazars is still under investigation with both leptonic (emission is produced by Compton scattering of synchrotron/external photons) and hadronic (emission is dominated by proton-produced synchrotron and hadronic cascades) models being considered. These models can be diagnosed with high-energy polarization, since hadronic models predict systematically higher degrees of X-ray and $\gamma$-ray polarization than leptonic ones [10]. Moreover, since the polarization signatures of radiation produced by synchrotron radiation are well understood, the transition from low-energy to high-energy polarization can be an important indication of the degree of order of the magnetic field in the high-energy emission region [11]. A summary on how high-energy polarization can be exploited in order to understand the key processes in blazar jet physics will be given in section 2 .

The general formalism for calculating high-energy polarization has been well established. Linear polarization arises from synchrotron radiation of relativistic charged particles in ordered magnetic fields, while Compton scattering of relativistic electrons will reduce the degree of polarization to 
about half of the target photon's polarization. The Compton (Klein-Nishina) cross section is generally polarization-dependent. Polarized photons scatter preferentially in a direction perpendicular to their electric field vector. Thus, when non-relativistic electrons scatter of an anisotropic radiation field, polarization can be induced even when the target photon field is unpolarized. Compton scattering by relativistic electrons induces no polarization since the target photon field is approximately axisymmetric around the electron momentum (in the electron rest frame), making any anisotropy of the target photon field irrelevant [1] [12].

In a model where thermal electrons, and a power-law tail of non-thermal electrons scatter an external radiation field, high-energy radiation results from scattering by relativistic electrons, and the radiation is predicted to be unpolarized. On the other hand, the low-energy radiation that results from scattering by thermal electrons, could be highly polarized. Monte Carlo methods are very flexible in the treatment of Compton scattering of arbitrary photon fields by arbitrary particle distributions and are therefore very useful for the investigation of Compton polarization, in order to investigate the transition from low-energy to high-energy polarization from anisotropic Compton scattering. The advantages of using Monte Carlo methods, as well as the treatment of polarized radiation in Monte Carlo codes, will be discussed in section 3.

\section{Scientific potential and model predictions}

In this section we aim to give a straightforward view of how high-energy polarization can address open questions surrounding blazar jets, rather than a detailed discussion of each model (a full review can be found in Böttcher [3]). We give a simple explanation of the problems yet to be fully understood, with single examples to illustrate how the polarization signatures change, depending on the model considered. In particular, we focus on the radiation mechanisms (leptonic or hadronic high-energy emission) in section 2.1, while in section 2.2 the mode of particle accelerations (shocks or magnetic reconnection) and the location of the most efficient energy dissipation site are discussed.

\subsection{Radiation mechanisms}

The SEDs of blazars exhibit two broadband peaks. The first (low-energy) peak is attributed to synchrotron emission from leptons, which gives a good explanation for the spectrum in terms of both the spectral shape and the polarization signatures. Observations of FSRQs and low-energy peaked BL Lac objects at low energies (radio to optical), confirm that the low-energy peak is polarized as expected for synchrotron radiation. The high-energy peaked BL Lac objects, however, have their first peak in the $X$-ray regime. $X$-ray polarimetry can therefore be used to confirm the synchrotron nature of this peak [10] [13] [14].

The origin of the second (high-energy) peak is less clear with the leptonic and hadronic models both consistent with the spectral shape of the SEDs [15]. Zhang and Böttcher [10] evaluated the expected high-energy polarization signatures in both models, demonstrating that the leptonic 
model predicts $X$-ray and $\gamma$-ray polarization degrees of $\Pi \leq 40 \%$, while the hadronic model predicts higher polarization degrees of $\Pi \leq 75 \%$ (see figure 1 ).

These predictions were made with the assumption of an ordered magnetic field, thus representing only upper limits on the actual expected polarization signatures from regions with partially ordered magnetic fields. The observed degree of polarization, in regions that can confidently be described by synchrotron emission, can thus be used to quantify the order of the magnetic field. The transition from low-energy to high-energy polarization can thus be used to distinguish leptonic from hadronic high-energy emission from blazars.

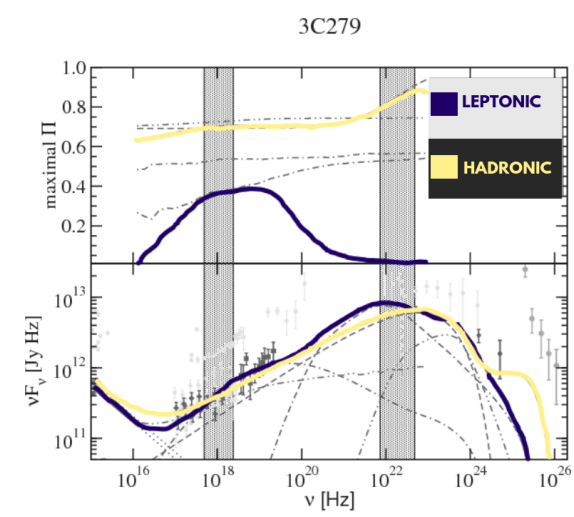

Figure 1: UV through $\gamma$-ray SED (lower panel) and the maximum degree of polarization (upper panel) for the FSRQ 3C279. The leptonic model fits are shown in blue and the hadronic model in yellow (from Zhang and Böttcher [10]).

\subsection{Particle acceleration}

Blazars show multi-wavelength variations which indicate strong particle acceleration. The highenergy emission in blazars likely originates in the most active acceleration regions [1]. Shocks and magnetic reconnection have been suggested as candidates for the particle acceleration mechanisms, both able to produce acceptable, power-law shaped, particle spectra. Shock models generally assume that the emission region has low magnetization, inferred from broadband SED modelling of blazars [15], with a significant amount of kinetic energy which can be converted into non-thermal particle energy through shock acceleration. A low magnetization is required for shocks being the dominant particle acceleration sites in blazars, since the presence of a dominant magnetic field suppresses efficient particle acceleration at strong shocks. Magnetic reconnection models accelerate the protons and electrons by converting the dominant magnetic field energy into particle kinetic energy in order to produce the non-thermal particle spectra observed [3][2].

Shock and magnetic reconnection scenarios have, nonetheless, different magnetic field configurations. Polarization properties of blazars indicate that the configuration of the magnetic field is not the same in newly injected material for different epochs. In shocks the magnetic field is 
compressed and its strength is, thus, increased, so that the flare may be accompanied by major polarization variations. In contrast to shocks, magnetic reconnection dissipates magnetic energy through the topological rearrangement of the magnetic field. The topological rearrangement of the magnetic field is, however, much faster than the global magnetic field diffusion time, and strong changes in the polarization are not expected during flares (see figure 2) [16][17]. The magnetic field evolution is also dependent on how strongly the jet is magnetized. It is, therefore, important to probe the characteristics of the energy in the jet, to determine whether the jet is dominated by kinetic or magnetic energy, and where most of the energy dissipates [3][2].

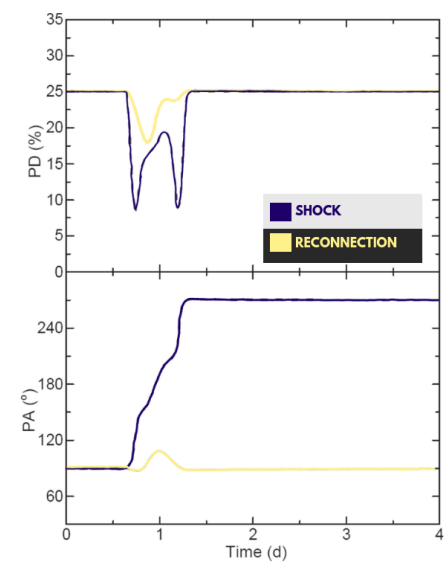

Figure 2: Polarization angle (lower panel) and polarization degree (upper panel) of shock (blue) and magnetic reconnection (yellow) scenarios (from Zhang [2]).

Time dependent polarization signatures of a kinetic-dominated and a magnetic-dominated jet emission environment are shown in figure 3. In a kinetic-dominated shock, the shock compression at the shock front can be very strong compared to the magnetic force. This causes the magnetic field to become highly ordered, along with strong changes in the polarization signatures, as well as high polarization degrees during flares. The magnetic field, strongly perturbed by the shock, will change considerably in the emission region and will, therefore, take a long time to recover to its initial, partially ordered state. As a result, the polarization signatures will also remain very high after the shock perturbation. Contrarily, in a magnetic-dominated shock, the shock is relatively weak compared to the magnetic force, and the magnetic field is not expected to change significantly during shock perturbation. Consequently, the polarization signatures stay approximately constant during flares, with a clear restoration phase of the magnetic field and polarization signatures at the end of the flare [2].

Optical polarization variations favor a magnetized jet since, according to optical polarization monitoring, the polarization degree is $\Pi \sim 10 \%$, with variable polarization signatures returning relatively quickly to the quiescent state. However, optical polarization is inconclusive with regard to the polarization properties in the regions with the most energetic particles. High-energy polarization can probe the most active acceleration regions and, as such, will be very useful to constrain the jet magnetization, and where most of the energy dissipates [3][18]. 


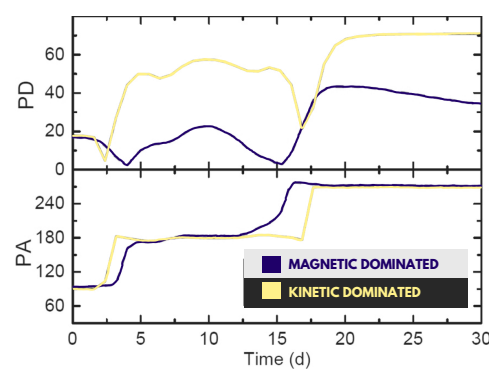

Figure 3: Polarization angle (lower panel) and polarization degree (upper panel) of kinetic dominated (yellow) and magnetic dominated (blue) jet emission environment (from Zhang [2]).

\section{Treatment of Compton polarization with Monte Carlo methods}

The Comptonization problem in astrophysics has been addressed in different ways. One method is to solve the nonlinear radiative transfer equations under certain approximations and assumptions, while using semi-analytical approaches for consecutive scattering orders. These methods have the advantages of being able to build a grid of solutions for a large range of free parameters in order to fit the data.

Another approach is the well known Monte Carlo method - a class of computational algorithms that rely on the randomness and repetitive nature of the process to produce numerical results invented by Ulam, von Neumann, and Metropolis [19]. Monte Carlo simulations consist of tracking photons in a geometrical setup, while recording the photons' history as they are transported through the computational domaine. The advantage of Monte Carlo methods lies in their generality; complex problems can be solved without simplifications in regard to the characteristics of the Comptonizing medium and the particle distributions (see Andreo [20] for a review). The first Monte Carlo simulations [21] were in good agreement with the semi-analytical approach, yet more rigorous, with significantly more computation time needed to achieve the required precision. However, the semi-analytic approach is less reliable for specific combinations of free parameters, and is a mono-dimensional description of the radiative process [22]. The stochastic nature of Monte Carlo simulations allows for a geometrical description of the radiative process, free from the restrictions of solving the radiative transfer equations. Notable work on the Monte Carlo approach to Comptonization is given by Pozdnyakov et al. in [23], [24], and Sunyaev and Titarchuk [25], as well as an extensive review by Pozdnyakov and Sobol [26]. Polarization of the radiation is, however, generally not included in these works, with the exception of Sunyaev and Titarchuk [25], who gave calculations on the degree of polarization in the Thomson regime.

More recently, in the view of future $X$-ray polarimetry missions, work on Monte Carlo methods includes calculations of the polarization signatures. These methods generally use photon packets that include the entire broad-band spectrum, to save computational time of the calculations. For example, the photon packet approach adopted by Schittman and Krolik [27] can mostly be used in 
the Thomson regime, and the energy exchange can be taken into account only by the boost from the emission frame to the reference frame of the electron, while the scattering is always elastic. Beheshtipour et al [28] expanded on the work of Krawczynski [29] and manage to include the proper Klein-Nishina treatment for Comptonization, as well as the contribution of non-thermal electrons in the emission region, while following the same photon packets approach as in Schittman and Krolik [27].

A new Monte Carlo code for Comptonization in Astrophysics (MoCA) was developed by Tamborra et al [30], using a single photon approach. The code is devoted to the spectrum and polarization signals in the $X$-ray band for accretion systems, in order to study the geometry of the corona, and better understand its origin. The single photon approach follows every photon individually from the source to the observer, instead of solving the radiative transfer equations. This enables the exploration of the whole space of free parameters that characterize the Comptonizing medium without any particular limitations. Following the treatment of Compton scattering of linearly polarized photons in Monte Carlo codes by Matt et al. [31], the contribution to the Stokes parameters is registered for every photon, which allows for the calculation of the photon's polarization degree and polarization angle. In this way, the polarization of the radiation can be obtained by the summation of the contributions of all the photons in a specified direction.

Monte Carlo methods can be used to simulate the anisotropic scattering by thermal electrons, with a power-law tail of non-thermal electrons, capitalizing on the method's inherent flexibility to study arbitrary distributions of soft target photons and electrons, both in terms of energy- and directional distributions, as well as the single photon approach's capability to delineate an emission region without specific limitations. Monte Carlo methods are, therefore, particularly favorable when considering the transition from low-energy to high-energy polarization in the SEDs of blazars.

\section{Conclusion}

The SEDs and variability of blazars can be modeled in many different ways, in particular concerning the production of $X$-rays and $\gamma$-rays, as well as the particle acceleration mechanisms. Polarization can be used as a powerful tool to distinguish between models, since the degree, orientation, and time variation of the polarization can provide more information for analysis. There is a renewed interest in high-energy polarization, since the predictions of high-energy polarization may be testable in the future with the anticipated $X$-ray and soft $\gamma$-ray polarimeters.

Monte Carlo simulations of Compton polarization form an adaptable approach to probe the transition between scattering of thermal and non-thermal electrons, and provide a way to make predictions of polarization signatures for various models. This reinforces the use of measurements of polarization signatures to suggest observational strategies in future projects - combining optical-, $X$-ray-, and $\gamma$-ray polarimetry signatures to determine the magnetic field's degree of order - and to use polarization as a diagnostic to distinguish between leptonic and hadronic high-energy emission from blazars. 


\section{References}

[1] M. Böttcher, D. E. Harris \& H. Krawczynski, Relativistic Jets from Active Galactic Nuclei, John Wiley and Sons, New Jersey 2012.

[2] H. Zhang, High-Energy Polarization: Scientific Potential and Model Predictions, Galaxies 5(3) (2017) 32 .

[3] M. Böttcher, Progress in Multiwavelength and Multi-Messenger Observations of Blazars and Theoretical Challenges, Galaxies in press

[4] N. Chakraborty, V. Pavlidou \& B.D. Fields, High energy polarization of blazars: detection prospects, APJ 798(1) (2014) 14.

[5] P. Soffitta, X. Barcons, R. Bellazzini, J. Braga, E. Costa, G.W. Fraser, S. Gburek, J. Huovelin, G. Matt, M. Pearce \& J. Poutanen, XIPE: the X-ray imaging polarimetry explorer, Experimental Astronomy 36(3) (2013) 523-567.

[6] R. B. Amini, Modern Assessment of the High-Energy Background Environment at Small Atmospheric Depths Using the X-Calibur X-Ray Polarimeter and Its Implications, Washington University in St. Louis, (Thesis - PhD) 2016.

[7] V. Tatischeff, M. Tavani, P. Von Ballmoos, L. Hanlon, U. Oberlack, A. Aboudan, A. Argan, D. Bernard, A. Brogna, A. Bulgarelli \& A. Bykov, The e-ASTROGAM gamma-ray space mission, in proceedings of Space Telescopes and Instrumentation 2016: Ultraviolet to Gamma Ray 9905: 99052N, (2016).

[8] M.C. Weisskopf, B. Ramsey, S.L. O’Dell, A. Tennant, R. Elsner, P. Soffita, R. Bellazzini, E. Costa, J. Kolodziejczak, V. Kaspi \& F. Mulieri, The imaging x-ray polarimetry explorer (IXPE), Results in Physics 06 (2016) 1179-1180.

[9] A. Moiseev, All-Sky Medium Energy Gamma-ray Observatory (AMEGO), in proceedings of ICRC2017, POS ( ICRC2017) 798 (2017).

[10] H. Zhang \& M. Böttcher, X-ray and gamma-ray polarization in leptonic and hadronic jet models of blazars, ApJ 774(1) (2013) 18.

[11] H. Zhang, X. Chen \& M. Böttcher, Synchrotron polarization in blazars, ApJ 789(1) (2014) 66.

[12] A. Celotti \& G. Matt, Polarization properties of synchrotron self-compton emission, MNRAS 268(2) (1994) 451-458.

[13] E.M. Rossi, D. Lazzati, J.D. Salmonson \& G. Ghisellini, The polarization of afterglow emission reveals $\hat{I} s ̧ \hat{A} \breve{A}$ ray bursts jet structure, MNRAS 354(1) (2004) 86-100.

[14] V. Pavlidou, E. Angelakis, I. Myserlis, D. Blinov, O.G. King, I. Papadakis, K. Tassis, T. Hovatta, B. Pazderska, E. Paleologou \& M. Baloković, The RoboPol optical polarization survey of gamma-ray-loud blazars, MNRAS 442(2) (2013) 1693-1705.

[15] M. Böttcher, A. Reimer, K. Sweeney \& A. Prakash, Leptonic and hadronic modeling of Fermi-detected blazars, ApJ 768(1) (2013) 54.

[16] L. Sironi, M. Petropoulou, \& D. Giannios, Relativistic jets shine through shocks or magnetic reconnection?, MNRAS 450(1) (2015) 183-191.

[17] E. G. Zweibel, \& M. Yamada, Perspectives on magnetic reconnection, Proc. Math. Phys. Eng. Sci. 472(2196) (2016) 20160479. 
[18] M. Böttcher, Spectral and Polarization Signatures of Relativistic Shocks in Blazars, Galaxies 4(3) (2016) 22.

[19] N. Metropolis, \& S. Ulam, The monte carlo method, J. Amer. Stat. 44(247) (1949) 335-341.

[20] P. Andreo, Monte Carlo techniques in medical radiation physics, Phys. Med. Biol. 36(7) (1991) 861.

[21] B. E. Stern, M.C. Begelman, M. Sikora \& R. Svensson, A large-particle Monte Carlo code for simulating non-linear high-energy processes near compact objects, MNRAS 272(2) (1995) 291-307.

[22] J. Poutanen \& R. Svensson, The two-phase pair corona model for active galactic nuclei and X-ray binaries: How to obtain exact solutions, ApJ 470 (1996) 249.

[23] L.A. Pozdnyakov, I.M. Sobol \& R.A. Syunyaev, Effect of multiple Compton scatterings on an X-ray emission spectrum-Calculations by the Monte Carlo method, Soviet Astronomy 21 (1977) 708-714.

[24] L.A. Pozdnyakov, I.M. Sobol \& R.A. Syunyaev, The profile evolution of X-ray spectral lines due to Comptonization-Monte Carlo computations, A\&A 75 (1979) 214-222.

[25] R. A. Sunyaev \& L.G. Titarchuk, Comptonization of low-frequency radiation in accretion disks Angular distribution and polarization of hard radiation, A\&A 143 (1984) 374-388.

[26] . L.A. Pozdnyakov, I.M. Sobol \& R.A. Syunyaev, Comptonization and the shaping of X-Ray source spectra: Monte Carlo calculations, A\&A Rev. 02 (1983) 189-331.

[27] J.D. Schnittman \& J.H. Krolik, X-ray Polarization from Accreting Black Holes: Coronal Emission, ApJ 712 (2010) 908-9241.

[28] B. Beheshtipour, H. Krawczynski \& J. Malzac, The X-Ray Polarization of the Accretion Disk Coronae of Active Galactic Nuclei, ApJ 850(1) (2017) 14.

[29] H. Krawczynski, Tests of general relativity in the strong-gravity regime based on X-ray spectropolarimetric observations of black holes in X-ray binaries, ApJ 754(2) (2012) 133.

[30] F. Tamborra, G. Matt, S. Bianchi \& M. Dovoiak, MoCA: A Monte Carlo code for Comptonisation in Astrophysics-I. Description of the code and first results, A\&A 619 (2018) A105.

[31] G. Matt, M. Feroci, M. Rapisarda \& E. Costa, Treatment of Compton scattering of linearly polarized photons in Monte Carlo codes, Radiat. Phys. Chem. 48(4) (1996) 403-411. 